\title{
What Levels of Guidance Promote Engaged Exploration with Interactive Simulations?
}

\author{
Wendy K. Adams, Archie Paulson and Carl E. Wieman* \\ Department of Physics, University of Colorado, Boulder, CO 80309 \\ *CWSEI, University of British Columbia, Vancouver, BC V6T 1 Z1
}

\begin{abstract}
We became teachers because we want everyone to be able to see through science the elegance in nature as we do. Our instincts and training may lead us to "tell” students about science and math as we understand it. Unfortunately research has shown that simply telling is not always the most effective way to share our understanding. Simulations are a valuable instructional resource and can provide a wealth of data about student engagement and learning. Approximately 250 interviews have been conducted with simulations developed by the Physics Education Technology (PhET) Project. We've conducted interviews using several different levels of guidance and found that the nature of guidance influences the amount of student engagement. Minimal but nonzero guidance with many of these simulations promotes optimum engaged exploration and learning.
\end{abstract}

Key Words: physics education research, learning, engagement, mental framework, simulations, PACS: 01.40.-d, 01.50.F-, 01.50.H-, 01.50.Lc, 01.40.gb

\section{INTRODUCTION}

Physics education researchers have found that prediction questions are necessary for students to gain a conceptual understanding from lecture demonstration. ${ }^{1}$ Crouch, et al. find that students learn little if anything from traditionally presented classroom demonstrations. Giving students a couple of minutes to predict the outcome and record their ideas increases student engagement and learning from demonstrations. Research on learning shows that it is necessary for students to construct their own understanding of scientific ideas within the framework of their existing knowledge ${ }^{2}$. To accomplish this process, students must be motivated to actively engage with the content and able to learn from that engagement. Prediction questions help the student identify what is important, and build a mental framework for examining the phenomena. Without this framework, there are too many details to for students to follow and remember.

In this paper we will discuss student interviews with interactive computer simulations. These interviews have shown that students learn more through self-guided engaged exploration which only occurs if the interviewer provides minimal to no guidance. This often goes against our instincts. Why does so little guidance work in this case? We believe there are two aspects. First, the simulation, through its structure and appearance, provides considerable implicit guidance. The second and more fundamental reason is related to the work on using innovation and then efficiency training ${ }^{3}$. Efficiency refers to teaching rote problem solving and focuses on making specific skills automatic. Innovation requires students to attempt to create their own solution to an unfamiliar problem and helps novices form a suitable mental framework. A good innovation task is balanced so that it is just outside the students' current knowledge but difficult enough they can't quite solve it on their own. This type of task includes contrasting cases and requires the students to investigate a problem in depth to determine what factors are important. Then, when a lecture or other instruction is provided, the students are more ready to learn. Schwartz, et al. ${ }^{3}$ have shown that the optimal corridor of instruction is to offer an Optimal Corridor of Instruction

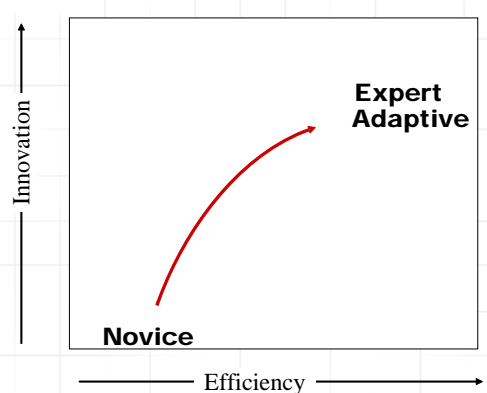

FIGURE 1: Innovation versus Efficiency from Schwartz, Bransford and Sears ${ }^{3}$. 
innovation task followed by efficiency instruction (FIGURE 1). If the students are told the answer before the innovation task, it "short circuits" the creation of a mental framework, resulting in poor conceptual understanding and transfer.

Innovation tasks, like prediction questions, help develop a framework for novices to store new information. The innovation tasks encourage novices to figure out on their own what types and pieces of information are needed for them to solve the task. Once this framework is constructed, information can be offered to the novice who is then more able to organize and store the information within the framework. This new framework may not be complete; however, due to the existence of a framework, the missing pieces can usually be fit into this new framework with only minor extensions.

\section{BACKGROUND}

The Physics Education Technology (PhET) Project ${ }^{4}$ has created 80 interactive simulations for physics and chemistry. These simulations have minimal text. One of the key features is the inclusion of balanced challenges such as little puzzles and clues. These challenges are attainable and slowly bring the student to the main goal of understanding the underlying scientific concept via the exploration of physical phenomena. The students interact with the simulations via engaged exploration where they can interact with this visual environment at their own pace, investigating what they are not sure about slowly and building an expert-like mental framework around the concept as they see what features affect how the simulation behaves. This creates an understanding that includes a visualization of the phenomena and many connections between the bits of knowledge.

As a part of the development of PhET simulations, we've video recorded over 250 think-aloud style interviews with more than 100 different student volunteers. The student is not asked for their opinion or feedback on the simulation during these interviews, only to think out-loud as they explore. Four to six $30-$ 60 minute interviews are conducted with each version of a simulation ${ }^{5}$. Interview results are used to modify the simulation if necessary and then a new series of interviews are conducted with new students. This process continues until the simulation is eliciting only correct concepts and the interface is intuitive to use.

The PhET simulations are very involved, interactive animated environments that create a unique opportunity for learning not only for the student but the researcher too. The simulations create common visualization for the researcher and the student so that the researcher can almost see what the student is thinking. If the student becomes quiet during the interview, the researcher can "see" what the student is thinking by watching what they are exploring with the simulation. The simulation also provides a common vocabulary. Students often use words they've gotten from the simulation or the researcher can watch what the student is using when they use a particular term to see more precisely what the student is thinking. If the student does not know what word to use for something, they simply demonstrate it with the simulation.

\section{LEVELS OF GUIDANCE}

We have conducted several different types of interviews with the PhET simulations. Here we define and compare four different levels of interview guidance:

Type A - no instruction,

Type $\mathrm{B}-$ driving questions,

Type $\mathrm{C}$ - gently guided, and

Type D - strongly guided.

For type A and B guidance, user learning is strongly dependent on the quality of the simulation. For Type $C$ and $\mathrm{D}$ guidance, user learning is generally independent of the simulation; however, Type $\mathrm{C}$ is very sensitive to the construction of the activity.

\section{Type A - No Instruction}

"Play with the simulation and talk out loud as you do things." When students explore a simulation with only these instructions, they play with many different things and start with eye catching and inviting items first. If the simulation is too complicated and/or too intimidating, students do not engage in exploration. If the simulation is not inviting and fun, they only play for a short bit or not at all. If the simulation is at a level that encourages exploration, students slowly learn about a phenomenon, discovering what factors make a difference and how they affect the phenomenon. Thus, students build a mental framework and fill in the information they've identified as important via their own questioning. Their interactions are highly dependent on the particular simulation. We have seen that the sequence of students' exploration depends on which features are most inviting and that some features are never noticed if the feature layout is poor or if students' attention is drawn somewhere else in the simulation. The number of controls and level of complexity of the simulation also influences how much the students learn from the simulation. With a welldesigned simulation, Type A guidance can be incredibly productive for learning; however, in the case of a poorly designed or highly complex simulation, this type of guidance can be a complete failure for certain students. 


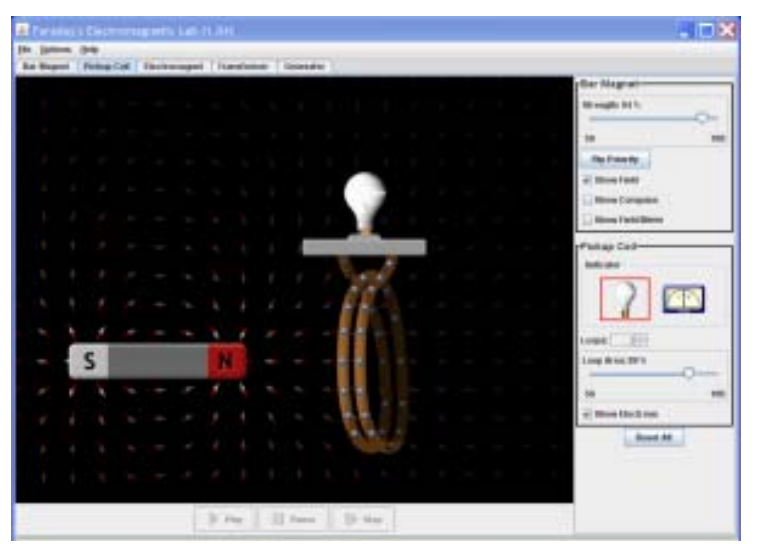

FIGURE 2: Faradays Electromagnetic lab. Students can explore magnets, electromagnets, a transformer and a generator.

\section{Type B - Driving Questions}

An interview with driving questions includes open, conceptual questions such as: "Can a magnet affect an electron?" and "What are some ways you can make a magnet?" These questions are asked before the student sees the simulation. After answering the questions, students are asked to play with the simulation and think out-loud as they do so.

When students explore a simulation with only these guiding questions, we observed them explore many different things, again choosing the most inviting items first. Similar to Type A interviews, during this engaged exploration, students build a mental framework and fill in the information they've identified as important via their own questioning. Typically about half of the interviewees spontaneously revise their answers to the driving questions as they explore, while the other half appear to have forgotten the questions. Again, if the simulation is too complicated or too intimidating, students do not spontaneously explore the simulation.

The driving questions provide some direction so that students explore the aspects of the simulation that we've asked them about - sometimes spontaneously and other times after we repeat the questions. A significant advantage of these driving questions is that, for some simulations, the students explore more deeply than they do with Type A guidance. For some simulations, this guidance is required in order to direct the students' attention to a specific concept. The disadvantage of driving questions is that it can often restrict student exploration. With Type B guidance, students tend to explore fewer features of the simulation than with Type A guidance, but they tend to explore particular areas in more depth. The driving questions that work best are open, conceptual questions that do not ask specifically about the simulation, but rather ask about the actual science concept.

Both Type A and Type B interviews result in very impressive student learning. As an example we will describe a typical interview with Faraday's Electromagnetic Lab (FIGURE 2) with students with no previous knowledge of the subject. All students work through the various tabs, typically taking just under an hour to do so. All students learn that electrons move only if the magnetic field is changing and that this movement lights the light bulb. If there are more loops of wire or if the area of the loops is larger, the bulb will be brighter. They also discover that a coil of wire attached to a battery has moving electrons that create a magnetic field that behaves exactly as the bar magnet does. Students are then able to successfully adjust all the items necessary to improve the efficiency of a generator ${ }^{6}$.

\section{Type C - Gently Guided}

Gently guided interviews include a series of questions along the lines of "In the 'Bar Magnet' tab, identify the things on the screen and in the controls in the control panel (at the right.) A. What does the 'Strength' slider do?, B. What does the 'Field Meter' do? etc..." With this sort of activity student exploration is limited to looking just enough into the specific aspect that has been asked about to answer the question. Then they wait for the next question. They rarely explore beyond the bounds of the question. This causes limited framework development because students are not asking their own questions. Often students are not able to tie the bits of information that they've learned together into complete ideas.

With this sort of guidance the responses are not as simulation dependent, since the students are not engaged in exploration. The one exception to this is when the simulation is exceptionally engaging - some students will go ahead and explore a bit beyond the particular question. Gently guided activities can be reasonably effective; however, only if very carefully designed through a cyclic process of writing, interviewing and then rewriting the activity until it takes the students to all of the particular aspects of the simulation they need to examine to understand the concept. We believe by adding and removing particular questions we can direct which aspects of the simulation the students notice. Paulson is currently conducting interviews to verify our hypothesis. The amount of learning with this type of gentle guidance is extremely sensitive to question choice, and so can have widely varying results.

\section{Type D - Strongly Guided}

This type of instruction can also be considered "cookbook". An example for the Faraday simulation would look like the following: "You are going to view the simulation Faraday's Electromagnetic Lab and will be asked a few questions about the concept of Faraday's Law and Flux. In this simulation, you can 
select any of the following options: a) Bar Magnet, b) Pick up Coil, c) Electromagnet, d) Transformer and e) Generator. At the right hand side of the screen you have a strength slider for the magnet, and options to turn the magnetic field indicators and the magnet on and off and to remove the compass or magnetic filed meter. While viewing the simulation, 1. Select the Bar Magnet tab. You can start moving the bar magnet around. Observe what is happening. Now describe what you see. 2. Is there any flux? If yes, why do you think so? Now select the Pick up coil tab..." We have observed many classes and labs using this type of guidance. With guided activities, very few students actually read everything written. They look to where the questions start and try to answer it. They only use the simulation when they can't answer a question without it, and then only enough to find the answer. There is no engaged exploration. In these cases, students develop only minimal parts of a knowledge framework. Giving specific instructions on how to use the simulation, results in a very different view of the simulation. The students appear to be afraid to try things on their own and will often ask what certain features do, without ever trying them on their own. This level of guidance seems to create a sort of barrier between the student and the simulation. The simulation belongs to the teacher and not the student. With this level of guidance, the quality of the simulation does not make a difference. The students are not exploring on their own or asking questions. These particular sorts of activities are not nearly as sensitive as type $C$ to question choice. They all result in the minimal understanding gained from typical cookbook activities.

\section{CONCLUSION}

We've found, through extensive simulation interviews, that exploration of the simulations under no guidance or with driving questions promotes students to explore the simulations where they gain physical insight into the phenomena via their own questioning. These results are consistent with the reasoning behind why innovation then efficiency is more effective at preparing students to learn. The innovation tasks force students to approach problem solving and knowledge acquisition in a similar fashion as experts - the task only provides enough information to create the framework of questions. If students are told the answers before the framework is developed, it short circuits this process and students do not develop a framework. Engaged exploration with simulations in the no guidance or driving question condition provides only enough guidance to require students to explore via their own questioning. Students form a mental framework through this process. Through its design (controls, features, visualizations), the simulation can influence this framework, shaping it into one that is similar to an expert's.

\section{FUTURE WORK}

These results are from several years of simulation development interviews. The PhET team is now embarking on several projects stemming from these results. First, as mentioned previously, the study of student response to gently guided interviews with strategically removed questions to see if students will limit their interaction to features mentioned in the guidance - features they always see when using Type A or B guidance. The second study will be to determine what are the crucial aspects of our simulations that make them effective for eliciting a deep understanding with Type A guidance. This will be a cyclic process where we test the aspects we've identified by modifying simulations that are not as effective in this sort environment until they become so. A third project is to learn how to structure homework and recitation materials to have just enough guidance to provide the structure to motivate students to engage in exploration via their own questioning.

\section{ACKNOWLEDGEMENTS}

We would like to thank Katherine Perkins and Noah Podolefsky for their many helpful discussions. This work would not have been possible without the expert simulations created by The PhET Team. PhET is supported by the National Science Foundation and the William and Flora Hewlett Foundation.

\section{REFERENCES}

1. e.g. Crouch, C. H., Fagen, A. P., Callan, J. P. and Mazur, E., Amer. J. Phys, 72, 2004, pp. 835-838.

2. e.g. Bransford, J., Brown, A., \& Cocking, R. (Eds.). How People Learn: Brain, Mind, Experience, and School., Washington, DC: National Academy Press, 2004.

3. Schwartz, D. L., Bransford, J. D. and Sears, D., "Efficiency and Innovation." Transfer of Learning from a Modern Multidisciplinary Perspective Jose Mestre (Ed). Information Age Publishing; North Carolina, 2005, pp. 1-52.

4. http://PhET.colorado.edu

5. Adams, W. K., Reid, S., LeMaster, R., McKagan, S. B., Perkins, K. K., \& Wieman, C. E., J. Interactive Learning Res., 12, 2008, pp. 397-419. http://phet.colorado.edu/research/index.php.

6. A sample transcript can be found at http://phet.colorado.edu/phetdist/publications/PERCinttranscript.html 